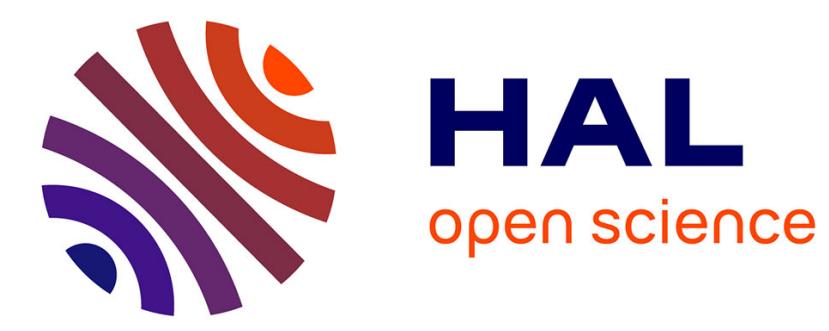

\title{
Turbulence properties in the cylinder wake at high Reynolds numbers
}

\author{
Marianna Braza, Rodolphe Perrin, Yannis Hoarau
}

\section{To cite this version:}

Marianna Braza, Rodolphe Perrin, Yannis Hoarau. Turbulence properties in the cylinder wake at high Reynolds numbers. Journal of Fluids and Structures, 2006, 22 (6-7), pp.757-771. 10.1016/j.jfluidstructs.2006.04.021 . hal-02545294

\section{HAL Id: hal-02545294 \\ https://hal.science/hal-02545294}

Submitted on 16 Apr 2020

HAL is a multi-disciplinary open access archive for the deposit and dissemination of scientific research documents, whether they are published or not. The documents may come from teaching and research institutions in France or abroad, or from public or private research centers.
L'archive ouverte pluridisciplinaire HAL, est destinée au dépôt et à la diffusion de documents scientifiques de niveau recherche, publiés ou non, émanant des établissements d'enseignement et de recherche français ou étrangers, des laboratoires publics ou privés. 


\title{
Turbulence properties in the cylinder wake at high Reynolds
}

\section{number}

\author{
M. Braza ${ }^{\mathrm{a}, *}$, R. Perrin ${ }^{\mathrm{a}}$, Y. Hoarau ${ }^{\mathrm{b}}$, \\ ${ }^{a}$ Institut de Mécanique des Fluides, UMR-CNRS-N ${ }^{\circ} 5502$, Av. du Prof. Camille Soula, 31400 Toulouse, France \\ ${ }^{\mathrm{b}}$ Institut de Mécanique des Fluides et des Solides, UMR-CNRS-ULP-N ${ }^{\circ} 7507$, Strasbourg, France
}

Received 9 October 2005; accepted 9 April 2006

\begin{abstract}
The present contribution analyses the turbulence properties in unsteady flows around bluff body wakes and provides a database for improvement and validation of turbulence models, concerning the present class of nonequilibrium flows. The flow around a circular cylinder with a low aspect ratio and a high blockage coefficient is investigated. This confined environment is used in order to allow direct comparisons with realisable 3-D NavierStokes computations avoiding 'infinite' conditions. The flow is investigated in the beginning of the critical regime at Reynolds number 140 000. The analysis is carried out by means of 2-D PIV, of 3-C PIV and of high-frequency 2D PIV. The experimental analysis contributes to confirm the validity of advanced statistical turbulence modelling for unsteady flows around bodies.

(c) 2006 Elsevier Ltd. All rights reserved.
\end{abstract}

\section{Introduction}

The accurate quantification of the turbulence properties in the near-wake region is of a priority interest concerning the physical analysis and the turbulence modelling of unsteady separated flows past bluff bodies. This

\footnotetext{
* Corresponding author.

E-mail address: braza@imft.fr.
} 
comprehension is a prerequisite for elaborating adapted and efficient turbulent modelling techniques for this category of flows characterised by a double physical nature, organised and chaotic.

The turbulence properties in the wake of a circular cylinder have been the object of a considerable number of experimental works during the last fifty years or over.

Concerning the measurement of near-wake turbulence by taking into account the impact of the coherent structures in the high-Reynolds number regime, the works of Cantwell (1975) and Cantwell and Coles (1983) have been a very important issue beyond the measurement techniques based on the statistical approach of turbulence, by using the phase-averaging concept and flying-hot wire anemometry. This allowed the measurement of turbulent quantities in a relatively near region, in connection with the influence of the coherent structure dynamics. These studies constituted a first ensemble of experimental results able to quantify this double physical nature of the present flow at the high Reynolds number regime. However, the limitations of flying hot-wire anemometry did not allow measurement in the vicinity of the wall, and the rather intrusive character of this measurement technique, comparing to optical ones like the LDV and the Particle Image Velocimetry (PIV), may affect the order of magnitude of the systematic errors in a general way.

Despite these limitations, the hot-wire technique, even using fixed probes, has been widely employed in connection with the phase-averaging concept in a number of experiments past bluff bodies, following the works of Cantwell and Coles (1983). Concerning the cylinder wake, Boisson (1982) and Boisson et al. (1983) carried out detailed measurements in the intermediate and far field, in the Reynolds number range of order (30 000-50 000). In these studies, the measurement of the phase-averaged velocities and turbulent stresses provided insight of the coherent part of the motion as well as the analysis of the intermittency phenomenon.

More recent experiments based on the analysis of the coherent part of the motion have been achieved by using multi-wire probes and appropriate signal processing techniques, giving access to three-dimensional aspects of the coherent motion. The three-dimensional nature of the intermediate wake region past the cylinder $(10<$ $x / D<40$ ) has been studied in detail in the work of Hayakawa and Hussain (1987), using X-wire rakes, allowing the quantification of the vorticity distribution in the spanwise and transverse planes at Re=13 000 . Significant three-dimensional effects on the dynamics of the coherent structures have been detected by means of spatial correlation and phase-aligned ensemble-averaging of the spanwise vorticity.

Concerning the influence of three-dimensionality in the experimental conditions, a number of studies have been devoted to the aspect ratio effects. In the work of Szepessy and Bearman (1991), a detailed study concerning the influence of this parameter has been carried out. It has been found that the aspect ratio mainly affects the fluctuation of the body forces and the pressure distribution for aspect ratio values varying from 1 to 12 and for the Reynolds number range $\left(8 \times 10^{3}-1.4 \times 10^{5}\right)$. A significant influence of the aspect ratio on the fluctuating lift coefficient has been found, especially for values of the aspect ratio lower than 4 . A considerable three-dimensional 
effect was detected in the phase of the vortex shedding and in the amplitude modulation of the lift signal at Reynolds number $1.3 \times 10^{5}$. This study is among the few ones investigating the turbulent wake past a cylinder at high Reynolds numbers and especially giving access to the pressure fluctuations. Furthermore, a thorough study of the aspect ratio influence on the base-pressure coefficient and on the Strouhal number has been carried out by Norberg (1995) in the Reynolds number range $\left(50-4 \times 10^{4}\right)$, reporting base suction measurements using a micromanometer. This study suggests the aspect ratio limits to provide independent conditions at mid-span versus the Reynolds number.

Recent works using Particle Image Velocimetry have been able to provide a refined view of the near-wake vortex dynamics and especially in respect of the longitudinal vortex structure. In this class of studies, the work of Lin et al. (1995) has shown the existence of longitudinal vortex structures at Re=10 000. Wu et al. (1996) have furnished a detailed structure of the von Kármán and longitudinal vortices in the near wake, beyond $x / D=2.6$, in the low Reynolds number range (Reynolds number of order 200 and 500).

Moreover, several spatial properties of the velocity structure functions in the near and intermediate wake are recently quantified by hot-wire measurements in the work of Gaudin et al. (1998) in the Reynolds number range (6 000-12 000). This work reports scaling laws for the structure functions based on selected position measurements in the wake. The indirect effect of the passage of the coherent structures is seen through the modification of the scaling exponents of the structure function in the near region, in relation to their values in the farther region and in respect of intermittence factor corrections.

The present nonexhaustive overview of the experimental studies devoted to the physical analysis of the turbulent wake around a cylinder has shown the strong impact of the coherent structure dynamics on the fine scale turbulence and the difference in the nature of the near-wake compared to the far-wake dynamics. The majority of these studies have provided analysis of the near region structure in the low Reynolds number range and of the intermediate and far region in the higher Reynolds number range. It appears that there is a need of a detailed study for the near-wake region in the high Reynolds number range, and this, by using a non-intrusive measurement technique. The importance of an accurate quantification of the turbulence properties in this region, mainly controlled by the unsteady separation and the formation of coherent structures, is capital for the comprehension of the physical mechanisms governing the flows past bluff bodies. This comprehension is a prerequisite for elaborating adapted and efficient turbulent modelling techniques for this category of flows, characterised by the mentioned double physical nature. Indeed, there are very few attempts of successful predictions of the turbulent flow past the circular cylinder at high Reynolds number, in the vicinity of the critical regime. The different approaches, either LES (still limited to the low Reynolds number regime for flows around bodies), or unsteady flow extensions of Reynolds averaged concepts ("Unsteady RANS") and DES (Detached Eddy Simulation), both more efficient for predictions of the high Reynolds number regimes, are not yet appropriately adapted in respect of the complexity of the present class 
of flows. This is due to the fact that the details of the near-region detached flow are not yet satisfactorily predicted. For these reasons, there is a need for improvement in the turbulence modelling approaches by means of well-focused data bases. These would aim at improving the above mentioned turbulence macro-simulation approaches. One of the objectives of the present study is to provide a refined data base with respect to the near-wake fields, allowing the access to key physical properties for the physical analysis and turbulence modelling. Having this objective in mind, the choice of the experimental parameters is done to facilitate the further task of prediction of this kind of turbulent flow in three dimensions and in high Reynolds number. To achieve this, it is preferable to have a reasonable size of the flow domain, to be able to simulate exactly the experimental conditions, rather than to be placed in the context of an 'infinite cylinder'. This would be unrealistic for high Reynolds number predictions in the state of the art. For these reasons, the blockage and aspect ratios chosen for the present study are significant parameters to take into account.

In this context, the main objectives of the present study are to provide a detailed cartography of the coherent structures in the near wake, subjected to the effects of the fine-scale turbulence. On this purpose, the 2-D PIV, the 3-C PIV and the high-frequency time-dependent 2-D PIV techniques have been used. The results have been processed, beyond the Reynolds averaging, by using the phase-averaging. The turbulence spectra in the field have been evaluated and decomposed in the coherent and incoherent parts, according to the Organised Eddy Simulation (OES) approach, Dervieux et al. (1998), concerning the turbulence modelling of this kind of flows. The prediction of this flow at high Reynolds number is performed by means of advanced statistical modelling in the context of OES. Discussion on the coherent, organised part of the flow as well as on the incoherent, turbulent part is addressed.

\section{Experimental set-up}

\subsection{Configuration}

The experiment has been carried out in the wind tunnel S1 of IMFT. The channel has a $670 x 670 \mathrm{~mm}^{2}$ square cross section. The cylinder spans the width of the channel and has a diameter of $140 \mathrm{~mm}$, giving an aspect ratio $L / D=4.8$ and a blockage coefficient $D / H=0.208$. The upstream velocity $U_{\text {inf }}$ at the centre of the channel is 15 $\mathrm{m} / \mathrm{s}$, so the Reynolds number based on the upstream velocity and the cylinder diameter $D$ is 140 000. Previous PIV measurements in the upstream region near the wall of the channel have shown that the velocity profile is uniform (at $0.5 \%$ at $x / D=-5$ up to a distance $9 \mathrm{~mm}$ to the wall. We have compared this value with the law of boundary layer Hinze $(1975): \delta / x=0.37\left(U_{0} x / \nu\right)^{-\frac{1}{5}}$ which gives $7.7 \mathrm{~mm}(0.055 D)$ at this abscissa. The free-stream turbulence intensity, measured by the hot-wire technique in the inlet was found $1.5 \%$. 


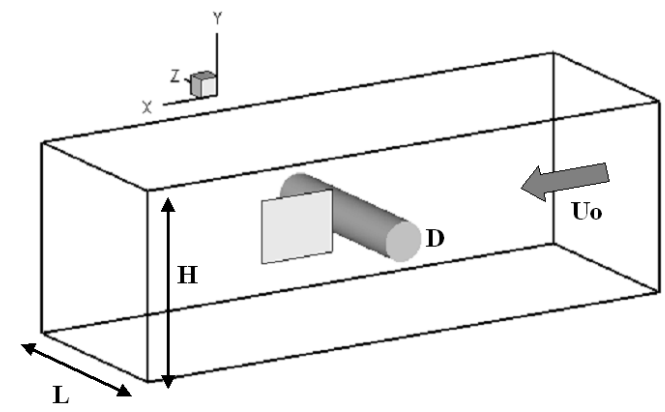

(a)

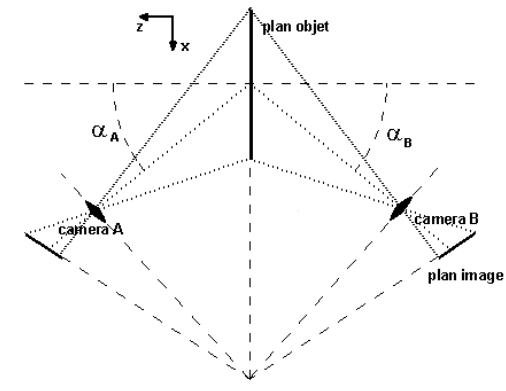

(b)

Fig. 1. (a) Flow configuration; (b) Scheimpflug configuration.

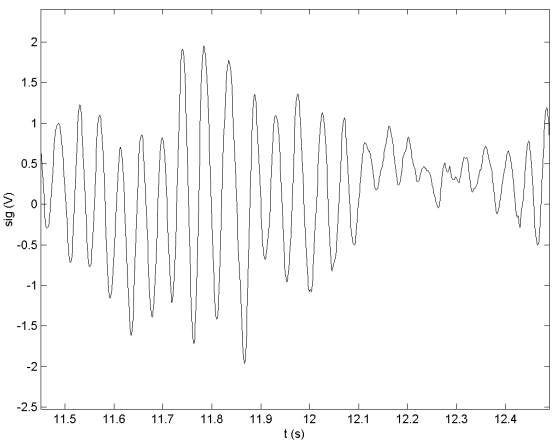

(a)

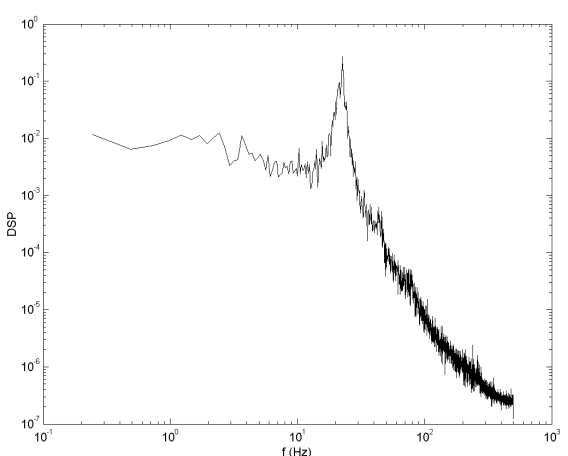

(b)

Fig. 2. (a) Wall pressure signal at $\theta=70^{\circ}$; (b) power spectrum.

All the quantities have been dimensionless by the $U_{\text {inf }}$ and $D$. This configuration is chosen for the purpose of allowing numerical prediction by using exactly the flow domain conditions and to avoid 'infinite-span' ones that demand a great deal of computational time in 3-D. The present configuration and data base have been used and are used in federative European programs in aeronautics: FLOMANIA (Flow Physics Modelling - an Integrated Approach) and DESIDER (Detached Eddy Simulation for Industrial Aerodynamics), both co-ordinated by EADS (European Aeronautics and Defense Systems).

\subsection{Measurements}

Wall pressure measurements have been carried out with a pressure transducer Validyne DP15-20. The frequency response is flat up to about $500 \mathrm{~Hz}$. The acquisitions have been achieved during 2 to $5 \mathrm{~min}$ at a sampling frequency of $1 \mathrm{kHz}$. The uncertainty on the $C_{p}$ coefficient is estimated to $3.5 \%$. 
Concerning the PIV measurements, a double-pulsed Nd-YAG laser Quantel $(2 \times 200 \mathrm{~mJ})$ and PCO-sensicam cameras (1280x1024 pixels) have been used. The seeding particles used have been DEHS (Di(2-ethylhexyl) Sebacate). A typical size of the particles is $1 \mu \mathrm{m}$. The system, both camera and laser, has operated at a frequency of $4 \mathrm{~Hz}$. The measurements have been carried out in the near wake of the cylinder in the $(x, y)$ plane located at the middle span position $z=0$ (Figure $1(\mathrm{a})$ ).

2- $C$ PIV: The camera was equipped with a $35 \mathrm{~mm}$ objective lens at a diaphragm aperture of 11 . The size of the measurement area has been $238 \times 188 \mathrm{~mm}(1.34 D \times 1.7 D)$. The software used to analyse images is a product of IMFT ("service signaux-images"). The algorithm is based on a 2-D FFT cross-correlation function implemented in an iterative scheme with a sub-pixel image deformation, according to Lecordier and Trinite (2003). The flow has been analysed by cross-correlating $50 \%$ overlapping windows of $32 \times 32$ pixels. This yielded fields of 77 x 61 vectors with a spatial resolution of $3.13 \mathrm{~mm}(0.0224 \mathrm{D})$. This resolution is proven to be sufficient for the evaluation of the major part of the turbulent stresses, according to tests that have been carried out by using smaller PIV planes and a calculus of vectors with an interrogation window of 16x16 pixels. Nevertheless, the smallest-scale turbulence beyond the above resolution cannot be provided in the energy spectrum, as is generally the case for any PIV experiment.

3-C PIV: Three-component measurements were carried out by using stereoscopic PIV, both to check the influence of the $w$ component normal to the plane on the results and to quantify the third normal Reynolds stresses $\overline{w^{2}}$. The angular configuration of Scheimpflug (Figure 1(b)) is employed with angles of order $20^{\circ}$ (limited by the tunnel geometry). Similarly to Willert (1997), the two cameras were placed on either side of the light sheet, thus both images are stretched identically and it is possible to view the same area by the two cameras. The procedure employed to calculate the 3 components is the same as in Cid et al. (2002). The cameras were equipped with a Scheimpflug adaptor designed by the LML (Laboratoire de Mécanique de Lille) and with a 35 $\mathrm{mm}$ objective lens at a diaphragm aperture of 11 . The mean spatial resolution is similar to the $2 \mathrm{C}$-component one. The domain of measurement is $0.6<x / D<2.28$ and $-0.7<y / D<0.52$.

High-frequency time-dependent 2-D PIV: The same experimental set-up as in the classic 2-D PIV has been employed. The Laser operated at a frequency of $1000 \mathrm{~Hz}$. The details of the experimental set-up are in Perrin (2005). The time-dependent PIV allowed evaluation of field-turbulence spectra in a plane having reduced dimensions compared to the classic 2-D PIV (Figures 12 and 13).

\subsection{Reynolds averaging}

About 3000 pairs of images were analysed to generate converged turbulence statistics with the 2-C PIV. The uncertainties are estimated, using a $95 \%$ confidence interval. They are 0.02 for $U, 0.03$ for $V$ mean components, and 0.015 for $\overline{u^{2}}, 0.02$ for $\overline{v^{2}}$ and 0.01 for $\overline{u v}$ correlations. The estimated uncertainty for the third normal Reynolds 


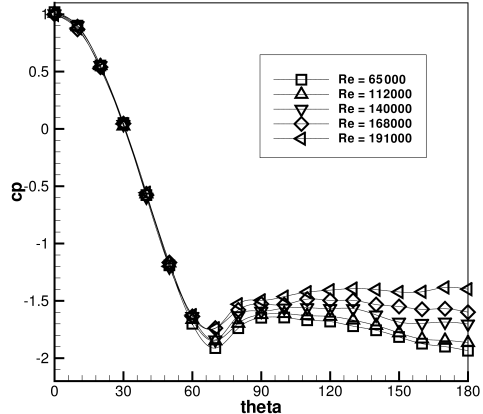

(a)

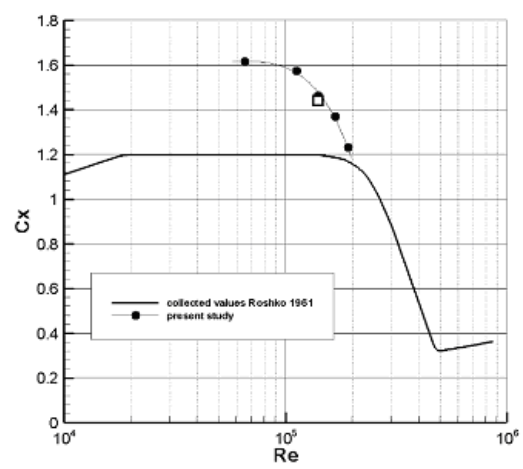

(b)

Fig. 3. (a) Mean pressure drag coefficient ; experimental Reynolds numbers according to the present study

65000

$\Delta 112000, \boldsymbol{\nabla} 140$ 000, 168000 and $\bullet 191$ 000. Comparison with the prediction at Re=140,000, $\square$, numerical value ; (b) Mean pressure drag coefficient versus Re.

stress $\overline{w^{2}}$ is 0.01 . For the 3-C PIV, 2570 instantaneous flow fields were acquired. The differences between the 2-C PIV and 3-C PIV results are lower than 0.03 for the mean components and lower than 0.04 for the correlations.

\subsection{Phase averaging}

The nearly periodic nature of the flow, due to the von Kármán vortices, allows the definition of a phase and the calculation of phase averaged quantities. The flow is classically decomposed into a mean component, a periodic fluctuation and a random fluctuation as $U_{i}=\overline{U_{i}}+\tilde{U}_{i}+u_{i}^{\prime}$, Reynolds and Hussain (1971). The phase average quantity is then $\left\langle U_{i}\right\rangle=\overline{U_{i}}+\tilde{U}_{i}$. The phase averaged quantities have been measured with the 2-D PIV technique.

The trigger signal used as an indicator of the vortex shedding is the pressure on the cylinder at an angle $\theta=70^{\circ}$ with the forward stagnation point. This location is near the separation and upstream the transition. Therefore, the signal has a strong quasi-periodic component at the Strouhal frequency, and it is not very affected by turbulent fluctuations. A typical pressure signal and its power spectrum are shown on Fig. 2. This exhibits a peak at the frequency $22.5 \mathrm{~Hz}$, corresponding to the vortex shedding at a dimensionless frequency (Strouhal number) $\mathrm{St}=f D / U=0.21$. The Strouhal number remains practically constant $(0.21)$ at the measured Reynolds from 65000 to 190 000. Both PIV images and pressure signal are acquired and stored to obtain phase averaged quantities. By post-processing the pressure signal, the phase is determined at each instant of acquisition of PIV. The flow fields are then ranged in 16 classes corresponding to phase angles dividing a period ; each class is in fact a window of width $2 \pi / 128$. Statistics are then performed in these classes. From the trigger signal, the phase of the flow is determined by using the Hilbert transform technique, as in Wlezien and Way (1979). The Hilbert transform allows calculation of the instantaneous envelope and phase from a band limited signal. Before applying the Hilbert 


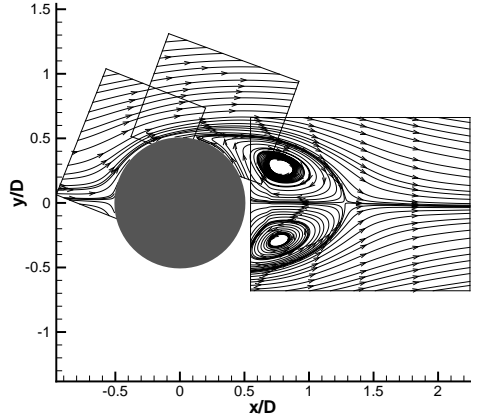

(a)

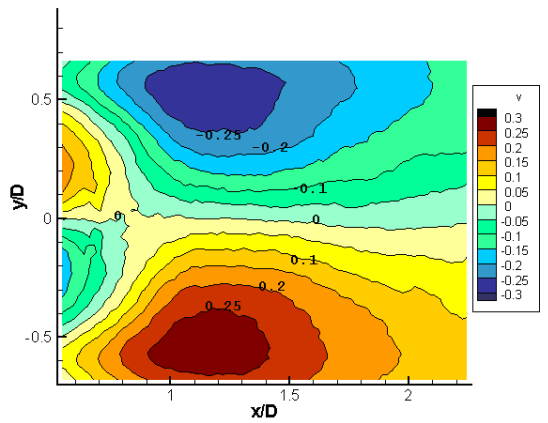

(c)

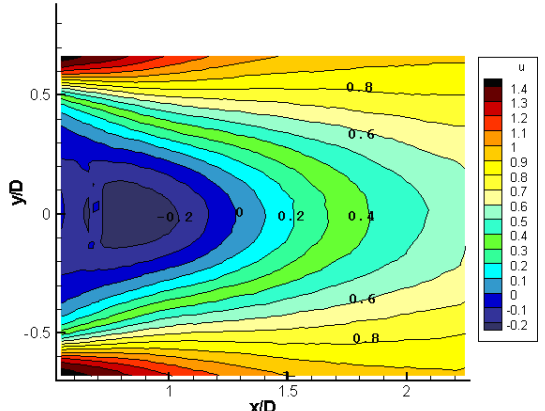

(b)

Fig. 4. Mean velocity field: (a) streamlines; (b) iso-contours of $\mathrm{U}$; (c) iso-contours of $V$.

techniques, a band-pass filter is applied to the signal. The instants where the phase cannot be determined because of the presence signal irregularities are detected and removed by a sort based on thresholds of amplitude and periods. The independence of the results from these parameters has been checked.

Finally, about 170 images are collected per class and averaged. The estimated uncertainties are 0.07 for $\langle U\rangle$, 0.1 for $\langle V\rangle, 0.05$ for $\left\langle u^{2}\right\rangle, 0.08$ for $\left\langle v^{2}\right\rangle, 0.04$ for $\langle u v\rangle$.

\section{Flow regime}

Measurements of mean wall pressure coefficient have been carried out in the median section $z / D=0$, around the cylinder, every $10^{\circ}$ for different Re numbers from 65000 to 191000 (Fig. 3(a)). The mean pressure drag coefficient is evaluated by integration of the pressure (Fig. 3(b)). The base-pressure coefficient, $\left(-C_{p b}\right)$ is found higher than in non-confined flow conditions, because of the blockage ratio. This yields a drag coefficient higher 


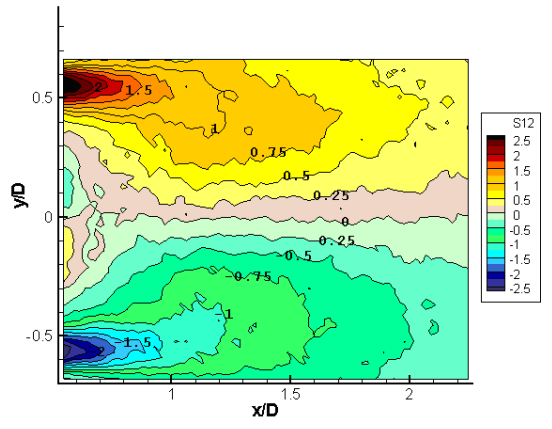

(a)

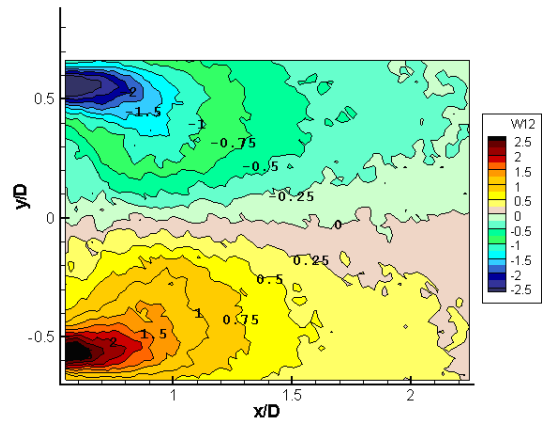

(b)

Fig. 5. (a) Mean strain rate $S_{12}$; (b) Mean rotation rate $\omega_{12}$.

than in a non-confined case. The drag decrease shows that the flow is at the beginning of the critical regime. This regime occurs at lower Reynolds number than reported in Roshko (1961), because of the free-stream turbulence intensity $(1.5 \%)$.

\section{Reynolds averaged fields}

The topology of the mean flow at $\mathrm{Re}=140000$ is studied in this section according to Reynolds averaging decomposition. Streamlines, iso- $U$ and iso- $V$ contours measured by 2-C PIV are shown in Fig. 4. Capital letters indicate Reynolds averaged quantities. As expected, a two symmetric eddies pattern is obtained, due to the averaging of the passage of the alternating vortices, resulting in a symmetric pattern for $U$ and in an antisymmetric one for $V$. As expected, the mean spanwise component $W$, measured by 3-C PIV is found to be null. The dimensionless recirculation length $l_{c}$ is found $1.28 \pm 0.03$ with 2-C PIV and $1.23 \pm 0.03$ with 3-C PIV. Values between 1.1 and 1.4 are found by several authors (Cantwell and Coles (1983), Norberg (1998), Djeridi et al. (2003)) in the same Re number range. In this last experiment, slight roughness in the cylinder surface provided a dispersion in the recirculation length. Given the difference in the boundary conditions (blockage, aspect ratio and inlet turbulence intensity) and experimental details having an important influence especially in the critical regime where the global parameters vary rapidly with Re number, there was a high sensitivity of the previous results in respect of the surface conditions. This problem was removed by having replaced the cylinder by a perfectly smoothed one and therefore a good repeatability of the measurements has been achieved. The mean velocity gradients, then the strain rate tensor $S$ and the rotation rate tensor $\omega$ are calculated with a central difference scheme. Fig. 5 shows the iso-contours of $S_{12}=\frac{1}{2}(\partial u / \partial y+\partial v / \partial x)$ and $\omega_{12}=\frac{1}{2}(\partial v / \partial x+\partial u / \partial y)$. As expected, a two lobes antisymetric configuration is obtained. The mean strain rate and the mean rotation rate are of the 


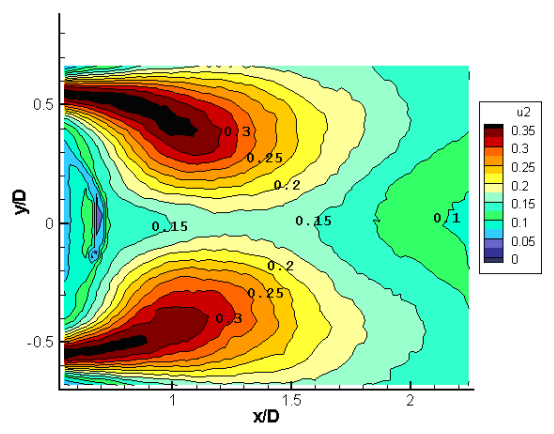

(a) $\overline{u^{2}}$

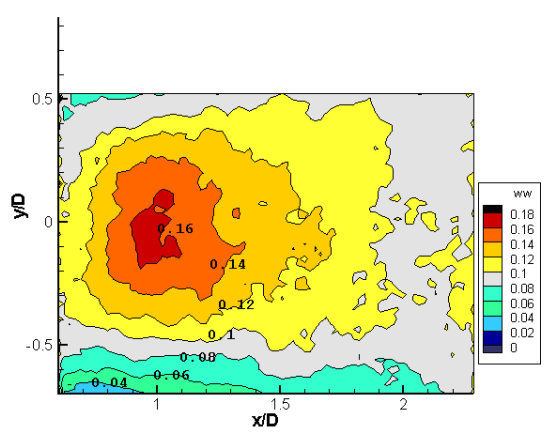

(c) $\overline{w^{2}}$

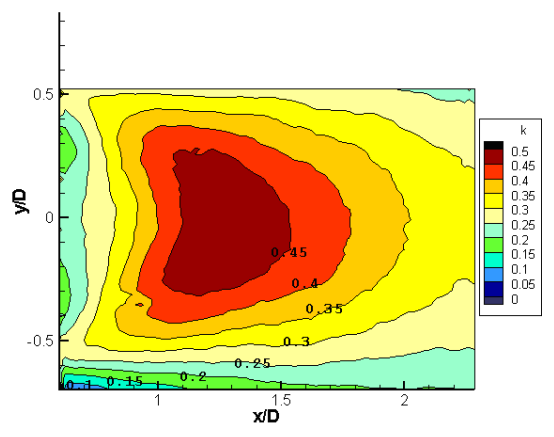

(e) $k$

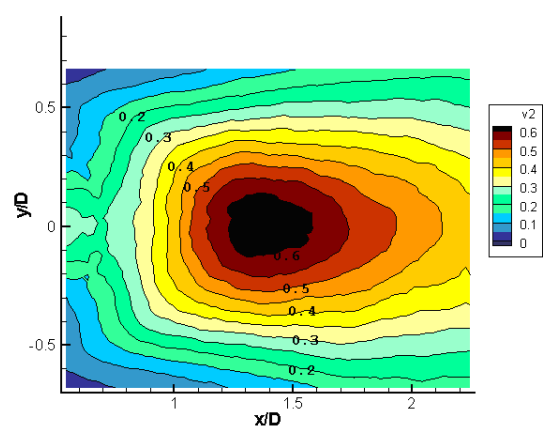

(b) $\overline{v^{2}}$

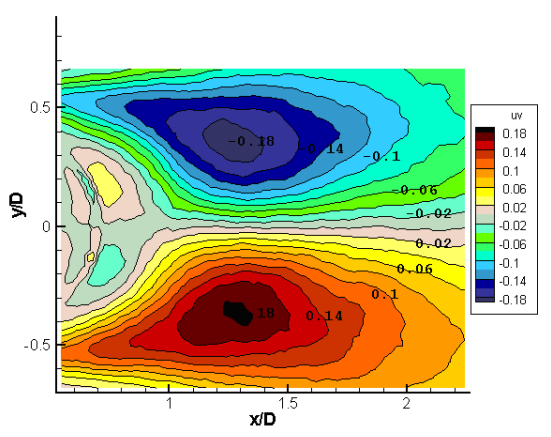

(d) $\overline{u v}$

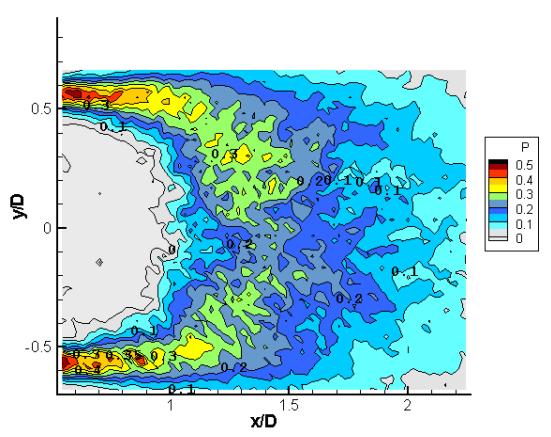

(df $P$

Fig. 6. (a-d) Mean Reynolds stresses; (e) mean turbulent kinetic energy; (f) production. 
same order of magnitude. The maximum of vorticity is found at the location $x / D=0.6$ and $y / D=0.55$. Figures 6(a)-(d) represents the iso-contours of the Reynolds stress tensor components. The $\overline{u^{2}}$ component has a two-lobe structure with maximum values located near $x / D=1$ and $y / D= \pm 0.5$. On the rear axis, the maximum value is found to be at $x / D=1.3$, that is close to the recirculation length; $\overline{v^{2}}$ has a one- lobe structure with the maximum value 0.6 on the rear axis at $x / D=1.4$. The 3 -C PIV precisely allows quantification of the $w$ component, that has been not measured up to now. This gives access to the complete turbulence stress tensor and to the complete kinetic energy and production terms, quantities that are very useful for modelling and less evaluated in 3-D to our knowledge. The $\overline{w^{2}}$ normal stress component presents also a one-lobe structure with lower value than the other normal stress. Its maximum value is found 0.18 at $x / D=1$, that is comparable with LDV measurement of Norberg (1998) in the rear axis. Concerning the shear stress field $\overline{u v}$, the maximum values $( \pm 0.2)$ are located on either side of the wake centre line at $x / D=1.4$ and $y / D= \pm 0.3$. As a classical result, $\overline{u w}$ and $\overline{v w}$ have been found null by 3-C PIV, confirming the two-dimensional character of the mean flow in the centre of the channel. As observed by many authors, it is noticeable that all the components have their maximum value near the vortex formation region. Then the turbulent kinetic energy, evaluated from the normal stresses, exhibits a one-lobe structure with the maximum located at $x / D=1.25$ (Figure $6(\mathrm{~d})$ ). The turbulent production term $P=-\overline{u_{i} u_{j}} \partial U_{i} / \partial x_{j}$ is evaluated and shown on Figure 6(e). Significant values coincide with the turbulent kinetic energy, but the maximum values are located near the shear layer at $x / D=0.6$ and $y / D= \pm 0.55$.

\section{Phase averaged fields}

The power spectrum of the wall pressure signal (Figure 2) exhibits a peak at the frequency $22.5 \mathrm{~Hz}$, corresponding to the vortex shedding at a dimensionless frequency (Strouhal number) $\mathrm{St}=f D / U=0.21$.

Fig. 7 shows the streamlines of the phase averaged flow at phase angles $0, \pi / 2$ and $\pi$. The periodic vortex shedding is clearly shown. The dimensionless vorticity is represented on Fig. 8 at four phase angles in the mean period. It is shown that the vorticity peak at the centre of a vortex decrease from 3 to 1 (in absolute value) when the vortex moves downstream from $x / D=0.6$ to $x / D=2$. Furthermore, the region of significant vorticity (taken arbitrarily to $\omega>0.5$ ) increases from a width of about $0.8 D$ to $1 D$. The vortex centres [identified with the $Q$ criterion, see Jeong and Hussain (1995)] have been marked for each phase angle and the mean trajectory is shown on Fig. 9. At $x / D=2$, the trajectories seem to be nearly parallel to the rear axis at a distance $y / D=0.25$, as found by Cantwell and Coles (1983) too. The longitudinal mean celerity of the vortices is evaluated by taking the derivative of the trajectories, to reach a value of $0.7 U_{\text {inf }}$ at $x / D=2$.

The Reynolds stresses at constant phase are evaluated. Fig. 10 shows the normal components $\left\langle u^{2}\right\rangle,\left\langle v^{2}\right\rangle$ and the shear stress $\langle u v\rangle$ at the phase angle $\varphi=\pi$. The general topology is found comparable to the result of Cantwell 


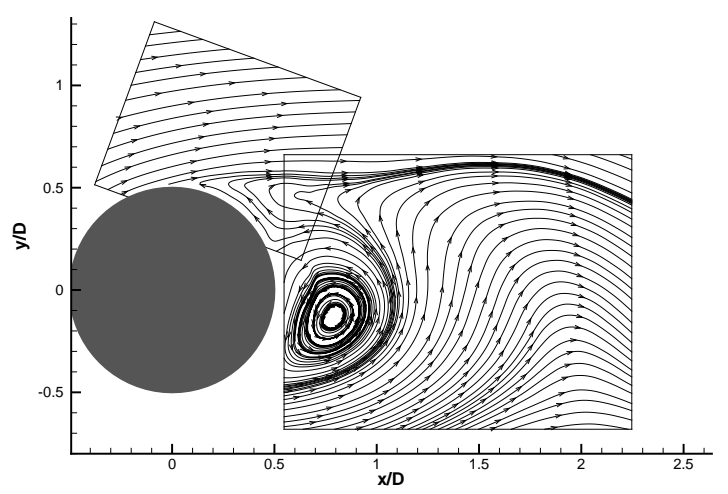

a) $\phi=0^{\circ}$

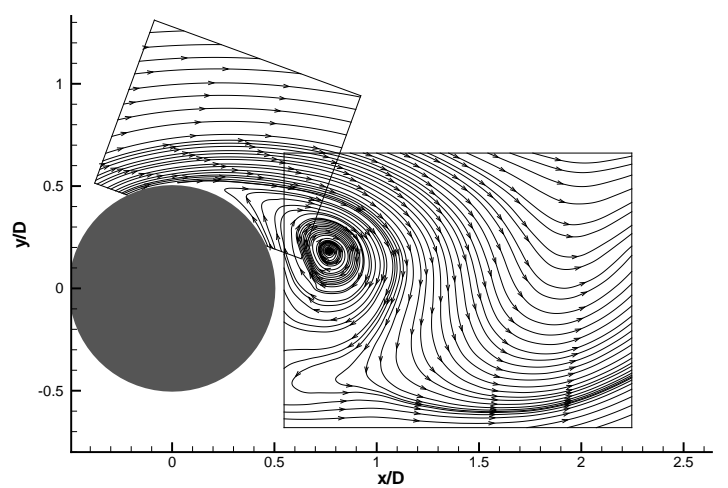

a) $\phi=180^{\circ}$

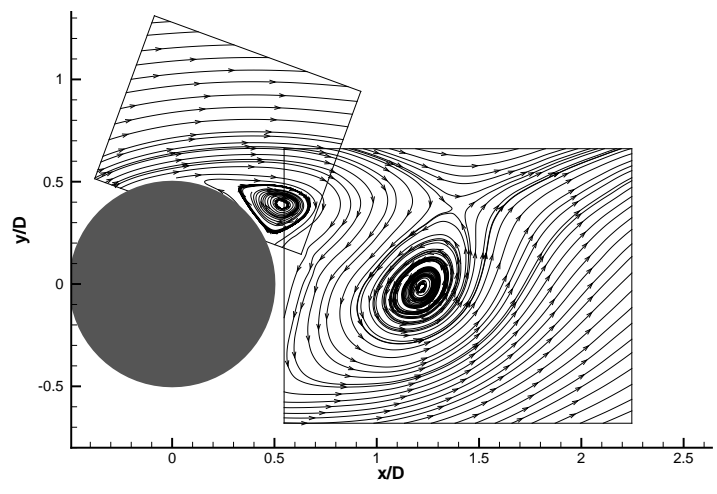

b) $\phi=90^{\circ}$

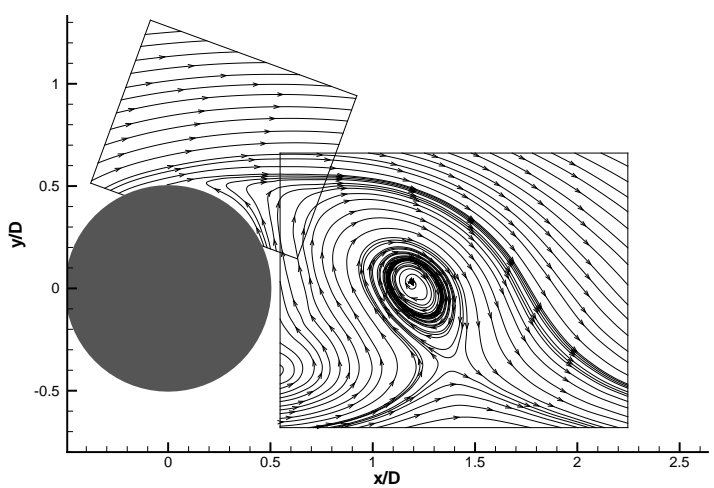

b) $\phi=270^{\circ}$

Fig. 7. Streamlines of the mean velocity fields at constant phase angles.

and Coles. This indicates that the normal stresses have high values near the centre of the vortices, while the maximum of the shear stress are located around the vortices. This is observed downstream of the formation region. In the formation region, significant values of $\left\langle u^{2}\right\rangle$ and $\langle u v\rangle$ are found in the shear layers. This topology is also found comparable to the results of Leder (1991) who measured phase averaged quantities in the near wake of a flat plate by LDV technique. The global Reynolds stress (in a statistical sense) can be decomposed into two contributions: the periodic motion and the random motion. The definition of this decomposition ensuring that the two contributions are uncorrelated, the Reynolds stresses can be expressed as $\overline{u_{i} u_{j}}=\overline{\tilde{u}_{i} \tilde{u}_{j}} \overline{\left\langle u_{i}^{\prime} u_{j}^{\prime}>\right.}$

Fig. 11 shows the two contributions of the shear stress. The topology and the level of them are comparable, the maximum values of the random motion being located considerably nearer the cylinder than the maximum value of the periodic motion. This behaviour was also indicated by Cantwell and Coles (1983) concerning the non-confined cylinder experiment. 


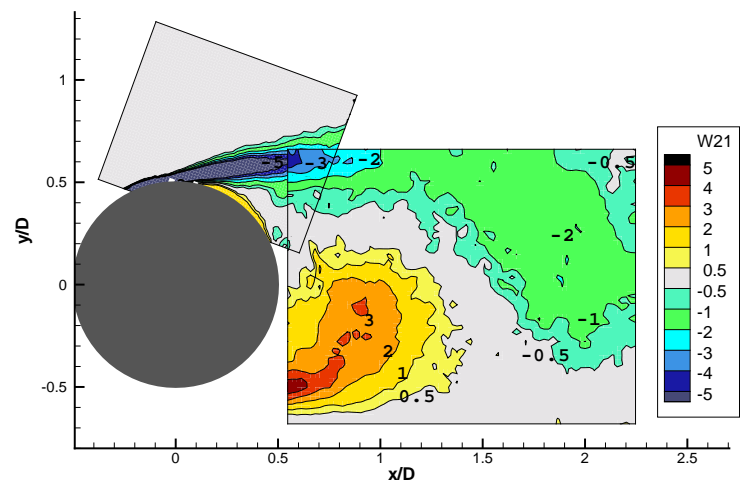

a) $\phi=0^{\circ}$

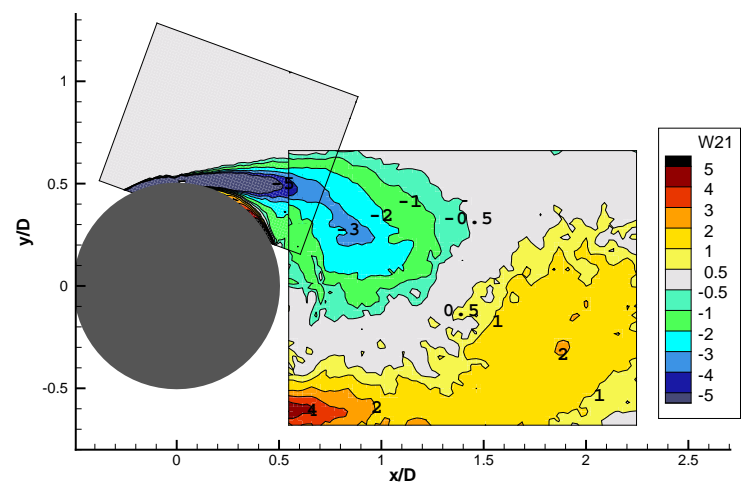

a) $\phi=180^{\circ}$

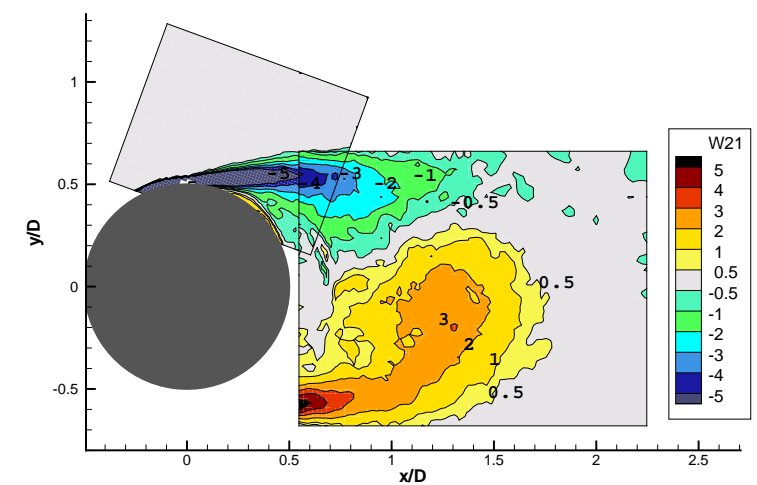

b) $\phi=90^{\circ}$

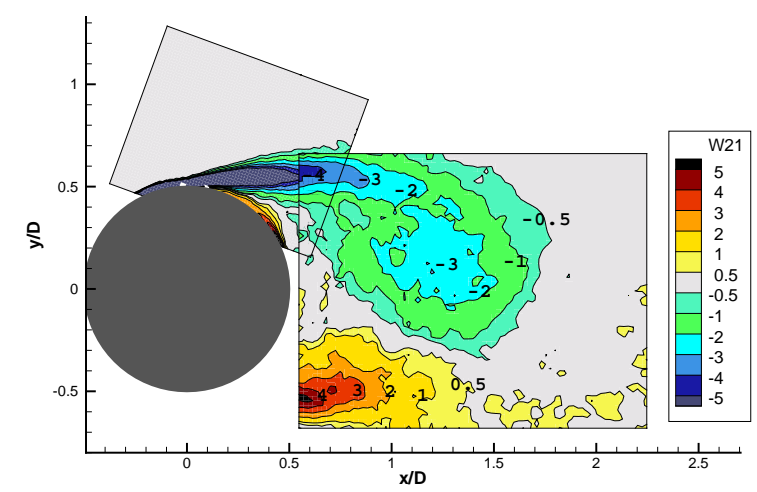

b) $\phi=270^{\circ}$

Fig. 8. Iso-contours of the mean shear-stress at constant phase.

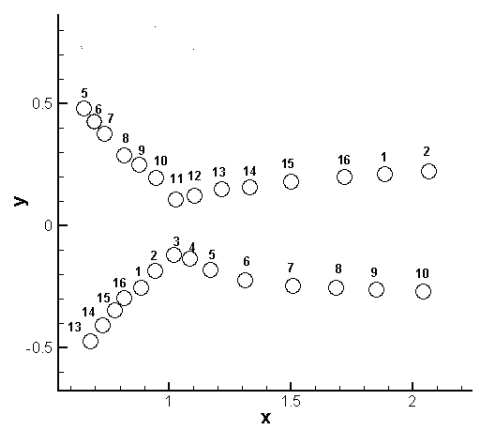

Fig. 9. Trajectories of the alternating vortices. Circles indicate the centres of the vortices and number indicates the phase $(1: \varphi=0 ; 2: \varphi=\ldots ; \ldots 16: 30 \pi / 16)$. 


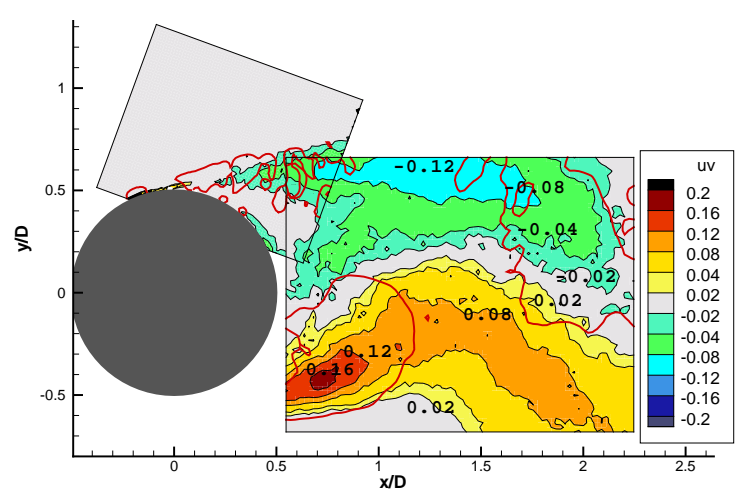

a) $\phi=0^{\circ}$

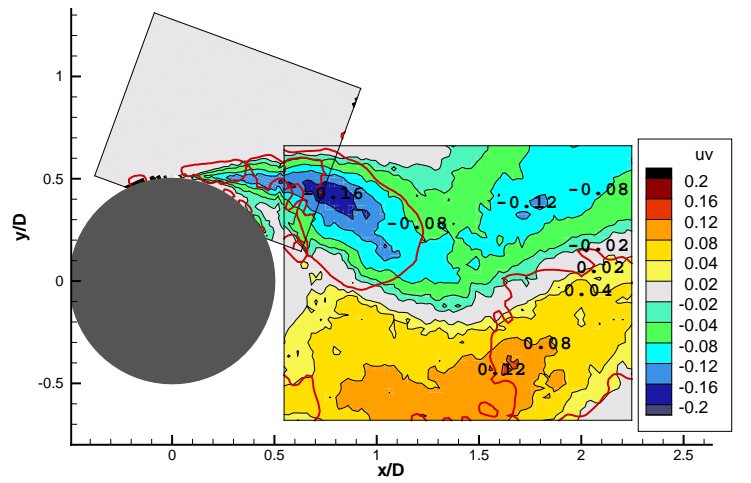

a) $\phi=180^{\circ}$

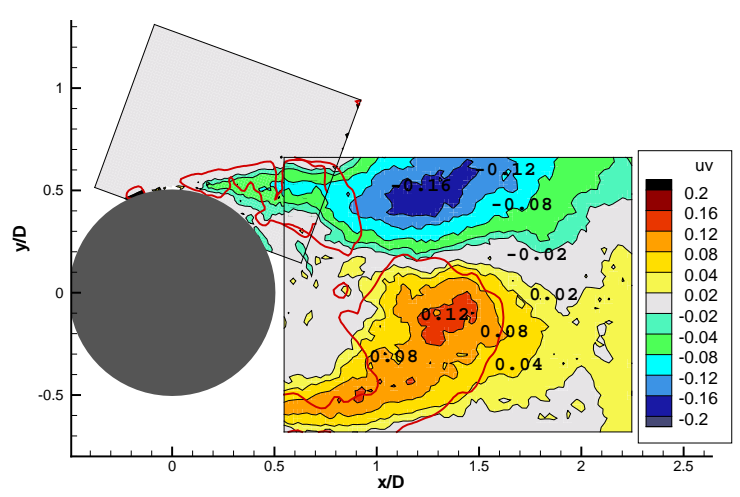

b) $\phi=90^{\circ}$

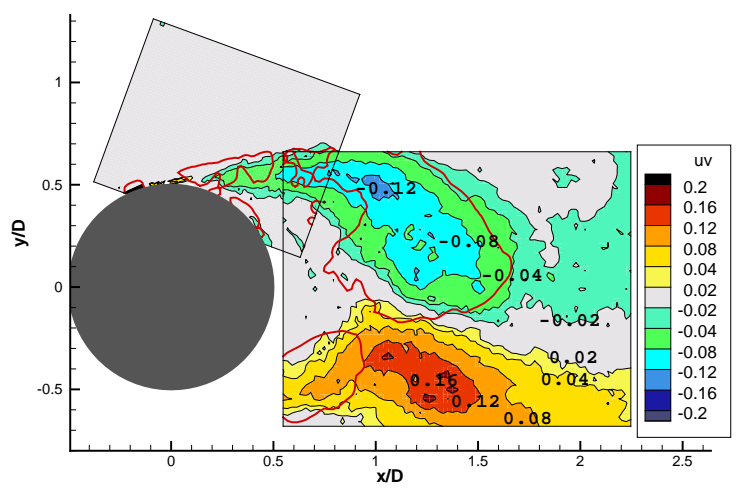

b) $\phi=270^{\circ}$

Fig. 10. Reynolds stresses at constant phase, red-line contours illustrate the topology of the alternating vortices

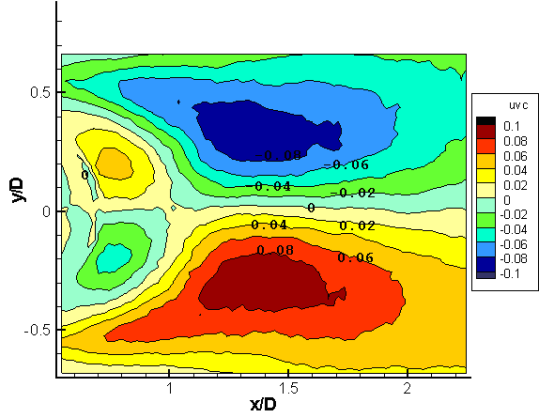

(a) $\overline{\tilde{u}_{i} \tilde{u}_{j}}$

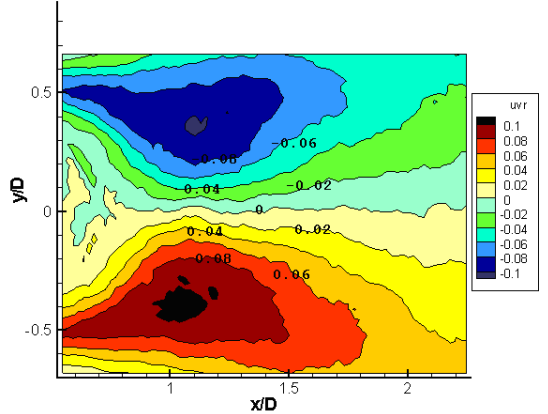

(b) $\overline{<u_{i}^{\prime} u_{j}^{\prime}>}$

Fig. 11. Shear stress: Contributions of the periodic motion and of the random motion. 


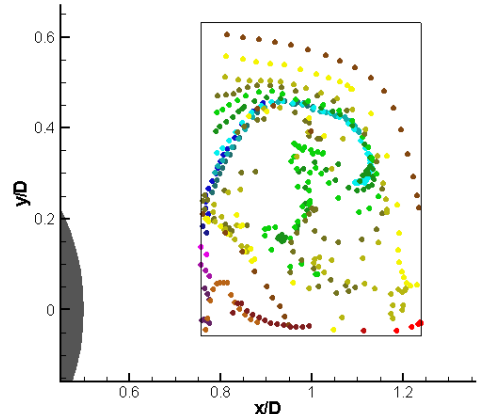

(a)

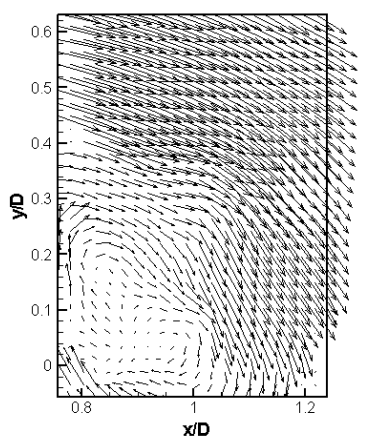

(b)

Fig. 12. Instantaneous streaklines and velocity field, time-resolved 2-D PIV.
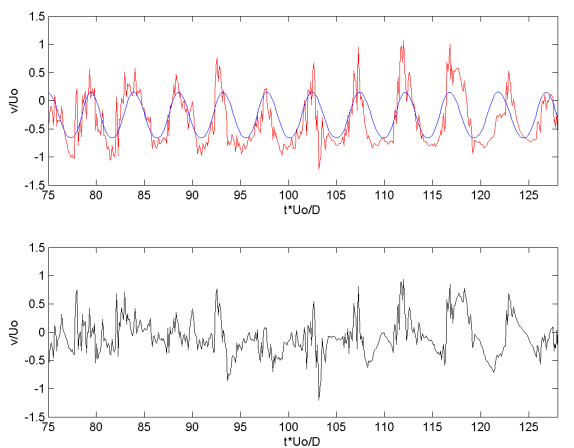

(a)

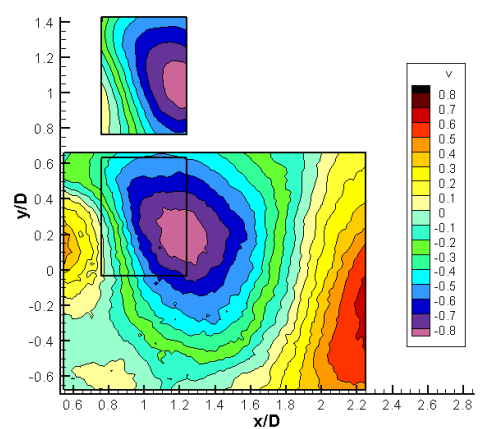

(b)

Fig. 13. (a) Time-dependent $V$-component signal at $x / D=1, y / D=0.5$; top: phase averaged signal (blue) is superimposed to the original signal (red), the turbulent fluctuation (bottom). (b) Time-dependent 2-D PIV phase-averaged $V$ component (small plan), compared with the classic 2-D PIV phase-averaging (larger plan)

\section{Time-dependent PIV fields}

The instantaneous velocity and streaklines, estimated from the time dependant PIV measurements by releasing particles along the left and bottom sides of the field are shown in Fig. 12. The recirculation mechanism as well as the turbulent mixing in the shearing regions within the vortex formation are clearly shown. The phase-averaging has been performed as described previously. Fig. 13(b) shows the iso- $\langle V\rangle$ phase-averaged velocity field (see plane above for the time-dependent PIV) in comparison with the classic 2-D PIV. A good comparison is achieved.

The time-dependent signal according to this technique is shown in Fig. 13(a), together with the phase-averaging and the turbulent fluctuation, according to the decomposition: $V$ (instantaneous) $=<V>$ (phase-averaging) + $v$ (fluctuation). Fig. 14 shows the corresponding energy spectra. It is shown that a residual peak stays in the so- 


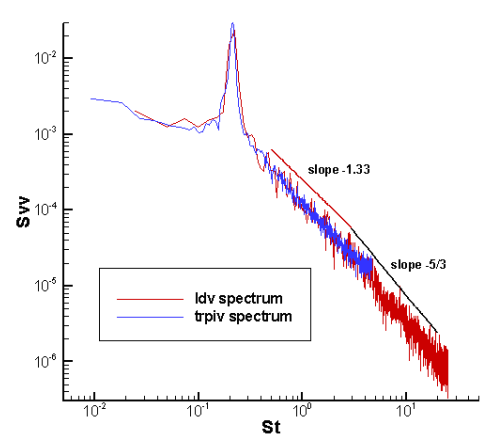

(a)

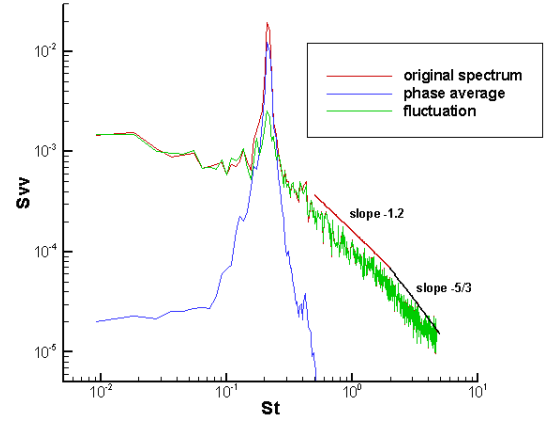

(b)

Fig. 14. a) Comparison between LDV and PIV spectra, $(x / D=1 y / D=0.375)$; b) PIV spectrum showing the slope modification in the inertial range, $(x / D=1 y / D=0.5)$, the phase-averaged decomposition of the energy spectrum as in OES (see fig. 15), $(x / D=1, y / D=0.5)$ and the fluctuation spectrum.

called 'random' part of the spectra. This is due to phase lag between the pressure signal and the velocity signal, occurring at some instant (as shown at $t \approx 1.1 \mathrm{~s}$ in Fig. 13). Therefore, as noticed by many authors (Cantwell and Coles (1983), Hayakawa and Hussain (1987), etc), the so-called 'random' component of the flow is due to both the small scale fluctuations and to this phase dispersion (phase jitter). Although not discussed in detail in this paper, the phase jitter effect has been analysed and alleviated using Proper Orthogonal Decomposition (Perrin $(2005))$.

\section{Flow Simulation}

In the context of the URANS/OES macrosimulation (Organised Eddy Simulation), Dervieux et al. (1998), the turbulent spectrum is decomposed in a first part regrouping all the coherent processes (resolved part) and in a second part regrouping all the chaotic processes independently on their size (spectrum to be modelled), as presented schematically in Fig. 15. It is recalled that in LES the distinction is done according to the structures size and this limits this approach to moderate Reynolds numbers concerning wall-turbulence around bodies. The fact that part of the spectrum to be modelled in OES is extended from the low to the high frequencies allows the use of statistical turbulence modelling, that is very efficient in high Reynolds number modelling of wall flows. In the time-domain, the equations are the phase-averaged Navier-Stokes equations, where the turbulent stresses have to be modelled by reconsidered statistical turbulence modelling closures. We had conjectured, Braza (2002), that due to the non linear interaction between the coherent part and the incoherent one, there must exist a shape and slope modification of the inertial part in the spectrum, in the vicinity of the peak. This has now been quantified, either 
by means of the LDV data or by the present study (time-dependent PIV data). Fig. 16 shows the cartography of the spectrum slope in the near wake. Especially the shear-layer regions are characterised by modified values comparing with the equilibrium turbulence value of $(-5 / 3=-1.667)$. The modification of the energy spectrum in the inertial range leads to modified turbulence scales in the context of the statistical two-equation modelling, as achieved in a previous work of us (Jin and Braza (1994), Hoarau et al. (2002), Bouhadji et al. (2002)), by means of the second-order moment closures. This yields a reconsideration of the eddy-diffusion coefficient for the class of the two-equation modelling, as well as improved damping functions to attenuate turbulence towards the wall, Jin and Braza (1994). In a companion paper of us, Hoarau et al. (2005), the details of the URANS/OES modelling are provided. In the present work, the prediction of the flow around the circular cylinder is presented by the modified URANS/k- $\varepsilon_{-}$OES modelling. Typical grids used are 400 x 100 x 100 . The boundary conditions are those of the above-mentioned physical experiment. In the outlet boundary downstream of the obstacle non-reflective boundary conditions are employed. A very good agreement in the prediction of the drag coefficient is achieved (Fig. 3(b)). It is well known that standard URANS models usually over-predict the drag coefficient [see collected works of the European program UNSI, unsteady viscous methods in the context of fluid-structure interaction, Haase et al. (2002)]. In Fig. 17(a), the time-dependent drag coefficient is presented. The Strouhal number is found to be 0.20 , quite close to the experimental value. In Fig. 18, the dynamics of the shear-layer eddies interacting with the adjacent von Kàrmàn eddies is shown. It is worthwhile noting that the standard URANS approaches attenuate the alternating eddies and are not able to allow formation of the Kelvin-Helmholtz vortices at this Reynolds number range. The alternating vortex pattern is in good agreement with the experiment concerning the expansion of the rotational area. In Fig. 17(b), the three-dimensional components of the vorticity are shown. The spanwise undulation due to the amplification of the secondary instability as well as the different classes of streamwise vortices are obtained.

\section{Conclusions}

The present study is an analysis of the coherent flow pattern and of the impact of the random turbulence in the flow past a circular cylinder at the beginning of the critical regime. The confined character of the flow allows further 3-D numerical simulations of the exact flow configuration, by using reasonable grid sizes comparing to non-confined experiments. The present study identifies the topology of the near-wake coherent structures and of the turbulent stresses according to the Reynolds-averaged and the phase-averaged decompositions. The timedependent PIV allowed dissociation of the coherent and incoherent parts in the way used for the turbulence modelling of strongly detached unsteady flows in the context of the Organised Eddy Simulation approach. It provides the modification of the energy spectrum in the inertial range, due to the non linear interaction between 


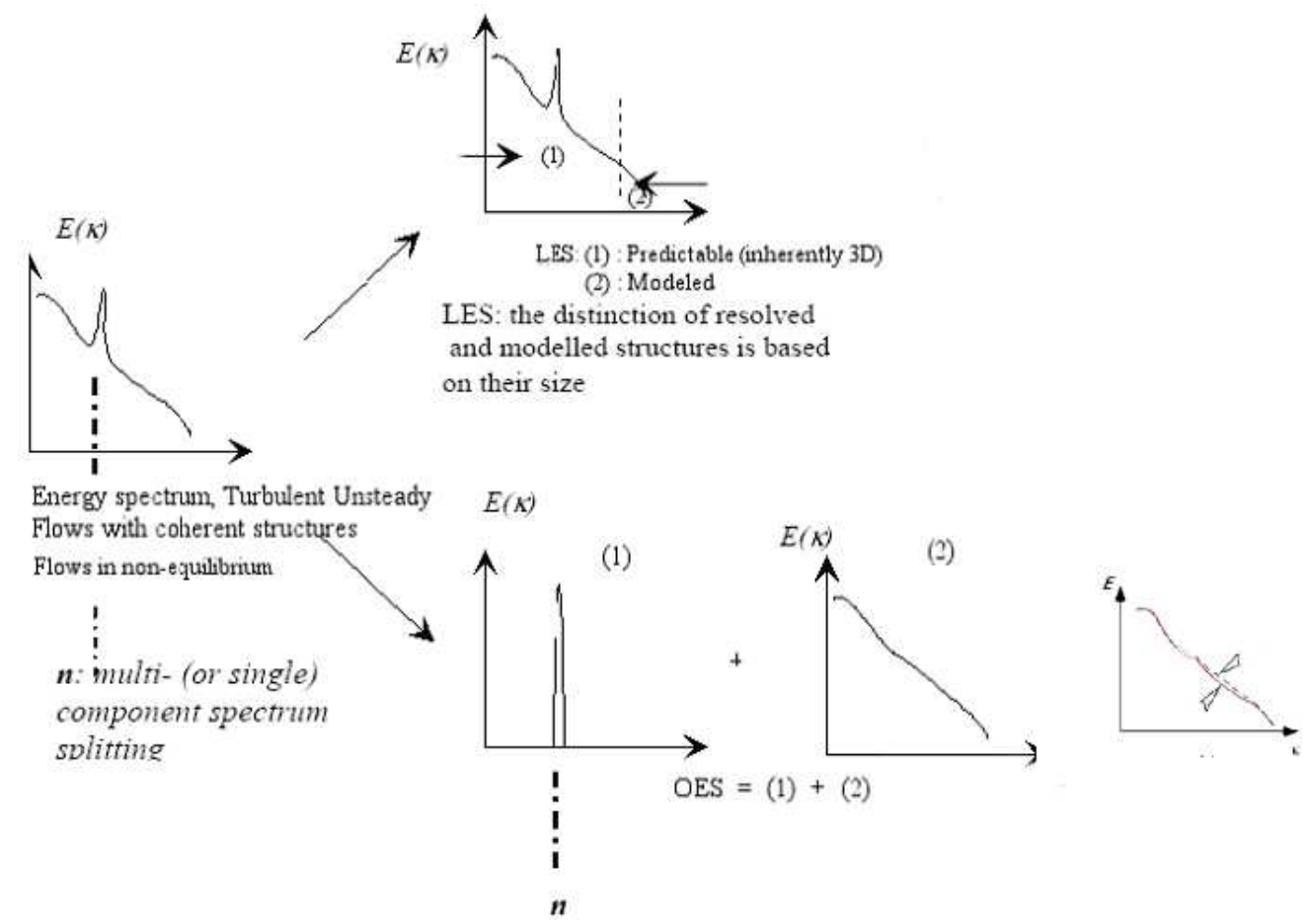

Fig. 15. Schematic representation of the energy spectrum in the URANS/Organised Eddy Simulation approach: the distinction between the structures to be resolved and those to be modelled is based upon their organised or random character. Part (2) of the non-equilibrium energy spectrum has to be modelled by reconsidered advanced statistical turbulence modelling, efficient in high-Re wall flows, due to the inertial-range modulation from equilibrium turbulence, schematically shown on the right.

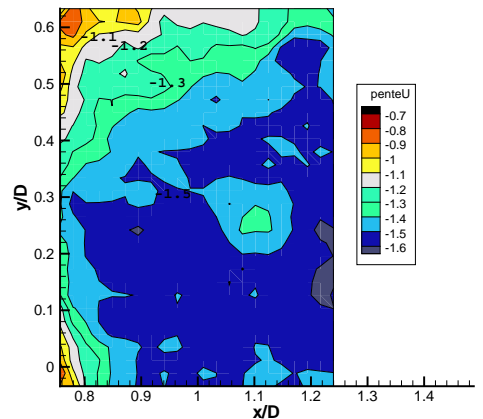

(a)

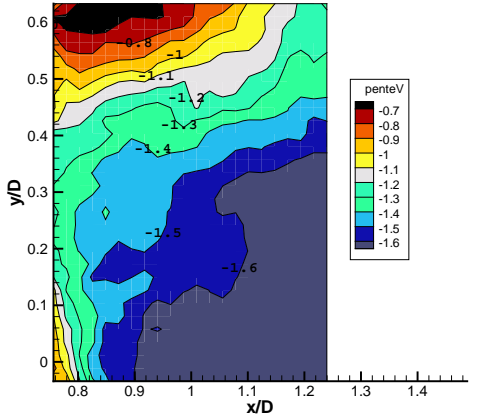

(b)

Fig. 16. Spectral slope modification in the inertial range by time-resolved 2-D PIV; topology in the near-wake according to: (a) $u$ component; (b) $v$ component 


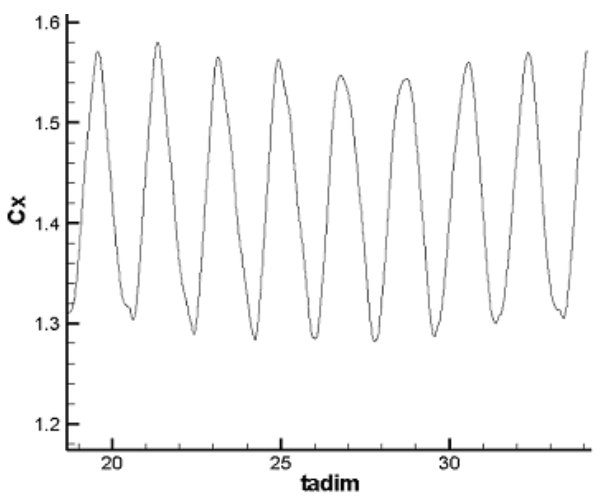

(a)

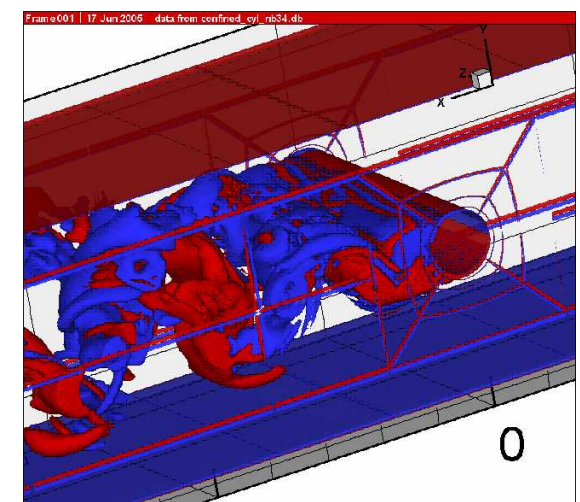

(b)

Fig. 17. (a) Time-dependent drag coefficient (prediction); (b) iso-vorticity surfaces $\omega_{z}$ and $\omega_{x}$, flow predicition at $\operatorname{Re}=140$ 000 at phase-angle 0.

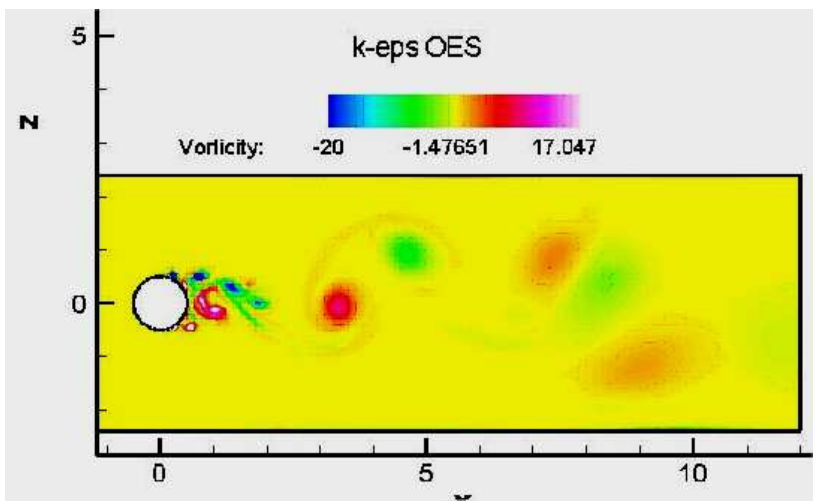

Fig. 18. Prediction of the flow at $\mathrm{Re}=140000$ with the URANS $/ k-\varepsilon-$ OES modelling. Instantaneous streamlines and iso-vorticity at the median section; formation and convection of the von Kàrmàn and Kelvin-Helmholtz eddies, phase-angle 180.

the coherent motion and the random turbulence. A successful prediction of this flow at $R e=140000$ is performed by the OES approach.

\section{Acknowledgement}

Part of the present study was carried out in the context of the European Commission Research Programme FLOMANIA G4RD-CT2001-00613 and DESIDER, N AST3-CT-2003-502842. The authors are grateful to E. Cid and S. Cazin of the "Signal and Image processing" general service of IMFT for having developed the experimental set-up for the PIV techniques, to F. Moradei for his contribution in the experimental set-up, and to A. Sevrain, P. Chassaing and G. Harran for their valuable scientific advice. 


\section{References}

Boisson, H., Chassaing, P., Ha-Minh, H., 1983. Conditional analysis of intermittency in the near wake of a circular cylinder. Physics of Fluids 26(3), 653-658.

Boisson, H. C., 1982. Développement de structures organisées turbulentes à travers de l'exemple du sillage d'un cylindre circulaire. Thèse d'État-ès- Sciences, de l'I.N.P. Toulouse, France.

Bouhadji, A., Bourdet, S., Braza, M., Hoarau, Y., Rodes, P., G.Tzabiras, 2002. Turbulence modelling of unsteady flows with a pronounced periodic character. Notes on Numerical Fluid Mechanics 81, 87-96.

Braza, M., 2002. Flow physics modelling in fluid-structure interaction. Notes on Numerical Fluid Mechanics, dedicated volume "Progress in Computational Flow-Structure Interaction", Sci. Eds. W. Haase, V. Selmin, B. Winzell, Publisher Springer 81, 145-154.

Cantwell, B., 1975. A flying hot wire study of the turbulent near wake of a circular cylinder at a reynolds number of 140000. Ph.D. Thesis, Cal Tech, Pasadena, CA.

Cantwell, B., Coles, D., 1983. An experimental study of entrainement and transport in the turbulent near wake of a circular cylinder. Journal of Fluid Mechanics 136, 321-374.

Cid, E., Cazin, S., Drouin, V., 2002. Validation de piv stéréoscopique et application a un écoulement aérodynamique de sillage 3d. In: $8^{\text {eme }}$ Congrès Francophone de Vélocimétrie Laser, Orsay, France.

Dervieux, A., Braza, M., Dussauge, J., 1998. Computation and comparison of efficient turbulence models for aeronautics. Notes on Numerical Fluid Mechanics, Vol 65, Ed. Vieweg.

Djeridi, H., Braza, M., Perrin, R., Harran, G., Cid, E., Cazin, S., 2003. Near-wake turbulence properties around a circular cylinder at high reynolds number. Journal of Flow Turbulence and Combustion 71, 19-34.

Gaudin, E., Protas, B., Goujon-Durand, S., Wojciechowski, J., Wesfreid, J., 1998. Spatial properties of velocity structure functions in turbulent wake flows. Physical Review 57, 59-78.

Haase, W., Selmin, V., Winzell, B., 2002. Progress in computational flow-structure interaction, results of the project UNSI, supported by the european union 1998 - 2000. Notes on Numerical Fluid Mechanics and Multidisciplinary Design 81.

Hayakawa, M., Hussain, F., 1987. Three-dimensionality of organised structures in a plane turbulent wake. Journal of Fluid Mechanics 206, 375-404.

Hinze, J., 1975. Turbulence. Mc Graw-Hill, New York.

Hoarau, Y., Braza, M., Revell, A., Laurence, D., Barthet, A., 2005. Physical analysis and modelling of turbulent unsteady flows around a wing. In: Proceedings International Symposium "Bluff Body Wakes and Vortex Induced Vibrations-4", June 2005, to be submitted in the Journal of Fluids and Structures.

Hoarau, Y., Favier, D., Braza, M., P.Rodes, G.Tzabiras, Allain, C., Berton, E., Maresca, C., 2002. Turbulence modelling of unsteady flows with a pronounced periodic character. In: IUTAM Symposium on Unsteady Separated Flows, 8-12 April 2002, Toulouse, France.

Jeong, J., Hussain, F., 1995. On the identification of a vortex. Journal of Fluid Mechanics 285, 69-94.

Jin, G., Braza, M., 1994. A two-equation turbulence model for unsteady separated flows around airfoils. AIAA Journal 32, $2316-2320$.

Lecordier, B., Trinite, M., 2003. Advanced piv algorithms with image distortion - validation and comparison from synthetic 
images of turbulent flow. In: PIV03 Symposium Busan, Korea.

Leder, A., 1991. Dynamics of fluid mixing in separated flows. Physics of Fluids 3, 1741-1748.

Lin, J., Vorobieff, P., Rockwell, D., 1995. Three-dimensional patterns of streamwise vorticity in the turbulent near wake of a cylinder. Journal of Fluids and Structures 9, 231-234.

Norberg, C., 1995. An experimental investigation of the flow around a circular cylinder: influence of aspect ratio. Journal of Fluid Mechanics 258, 287-316.

Norberg, C., 1998. LDV-measurements in the near wake of a circular cylinder. In: Conference on Bluff Body Wakes and Vortex Induced Vibrations Presented at ASME Fluids Engineering Division (Annual Summer Meeting), Washington D.C. pp. Paper 42 (FEDSM98-5202).

Perrin, R., 2005. Analyse physique et modélisation d'écoulements incompressibles instationnaires turbulents autour d'un cylindre circulaire à grand nombre de reynolds. Thèse de Doctorat, Institut National Polytechnique de Toulouse, France.

Reynolds, W., Hussain, A., 1971. The mechanism of an organized wave in turbulent shear flow - part 3 : Theorical models and comparison with experiments. Journal of Fluid Mechanics 54, 263-288.

Roshko, A., 1961. Experiments on the flow past a circular cylinder at very high reynolds number. Journal of Fluid Mechanics $10,345-356$.

Szepessy, S., Bearman, P., 1991. Aspect ratio and end plate effects on vortex shedding from a circular cylinder. Journal of Fluid Mechanics 234, 191-217.

Willert, C., 1997. Stereoscopic digital particle image velocimetry for application in wind tunnel flows. Meas. Sci. Tech. 8, 1465-149.

Wlezien, R., Way, J., 1979. Tecniques for the experimental investigation of the near wake of a circular cylinder. AIAA Journal 17:6, 563-570.

Wu, J., Sheridan, J., Welsh, M., Hourigan, K., 1996. Three-dimensional vortex structures in a cylinder wake. Journal of

Fluid Mechanics 312, 201-222.

\section{List of Figures}

$1 \quad$ (a) Flow configuration; (b) Scheimpflug configuration.

2 (a) Wall pressure signal at $\theta=70^{\circ}$; (b) power spectrum.

3 (a) Mean pressure drag coefficient ; experimental Reynolds numbers according to the present study $\bullet$, $\mathbf{\square} 65$ 000, $\Delta 112000, \boldsymbol{\nabla} 140$ 000, 168000 and $\bullet 191000$. Comparison with the prediction at Re=140,000, $\square$, numerical value ; (b) Mean pressure drag coefficient versus Re.

$4 \quad$ Mean velocity field: (a) streamlines; (b) iso-contours of $\mathrm{U}$; (c) iso-contours of $V$. 8

$5 \quad$ (a) Mean strain rate $S_{12} ;$ (b) Mean rotation rate $\omega_{12}$. 9

6 (a-d) Mean Reynolds stresses; (e) mean turbulent kinetic energy; (f) production. 10

$7 \quad$ Streamlines of the mean velocity fields at constant phase angles. 12

$8 \quad$ Iso-contours of the mean shear-stress at constant phase. 13 
9 Trajectories of the alternating vortices. Circles indicate the centres of the vortices and number indicates the phase $(1: \varphi=0 ; 2: \varphi=\ldots ; \ldots 16: 30 \pi / 16)$.

Reynolds stresses at constant phase, red-line contours illustrate the topology of the alternating vortices

11 Shear stress: Contributions of the periodic motion and of the random motion.

12 Instantaneous streaklines and velocity field, time-resolved 2-D PIV.

13 (a) Time-dependent $V$-component signal at $x / D=1, y / D=0.5$; top: phase averaged signal (blue) is superimposed to the original signal (red), the turbulent fluctuation (bottom). (b) Time-dependent 2-D PIV phase-averaged $V$ component (small plan), compared with the classic 2-D PIV phase-averaging (larger plan)

14 a) Comparison between LDV and PIV spectra, $(x / D=1 y / D=0.375)$; b) PIV spectrum showing the slope modification in the inertial range, $(x / D=1 y / D=0.5)$, the phase-averaged decomposition of the energy spectrum as in OES (see fig. 15), $(x / D=1, y / D=0.5)$ and the fluctuation spectrum.

15 Schematic representation of the energy spectrum in the URANS/Organised Eddy Simulation approach: the distinction between the structures to be resolved and those to be modelled is based upon their organised or random character. Part (2) of the non-equilibrium energy spectrum has to be modelled by reconsidered advanced statistical turbulence modelling, efficient in high-Re wall flows, due to the inertial-range modulation from equilibrium turbulence, schematically shown on the right. Spectral slope modification in the inertial range by time-resolved 2-D PIV; topology in the near-wake according to: (a) $u$ component; (b) $v$ component

17 (a) Time-dependent drag coefficient (prediction); (b) iso-vorticity surfaces $\omega_{z}$ and $\omega_{x}$, flow predicition at Re $=140000$ at phase-angle 0 .

18 Prediction of the flow at $\operatorname{Re}=140000$ with the URANS $/ k-\varepsilon$-OES modelling. Instantaneous streamlines and iso-vorticity at the median section; formation and convection of the von Kàrmàn and Kelvin-Helmholtz eddies, phase-angle 180. 